\title{
Electron Capture, Collision-Induced, and Electron Capture-Collision Induced Dissociation in Q-TOF
}

\author{
Valery G. Voinov, ${ }^{1,2}$ Max L. Deinzer, ${ }^{1}$ Joseph S. Beckman, ${ }^{1}$ Douglas F. Barofsky ${ }^{1}$ \\ ${ }^{1}$ Department of Chemistry, Oregon State University, Corvallis, OR, 97331, USA \\ ${ }^{2}$ Pacific Institute of Bioorganic Chemistry, Vladivostok, Russia
}

\begin{abstract}
Recently, we demonstrated that a radio-frequency-free electromagnetostatic (rf-free EMS) cell could be retrofitted into a triple quad mass spectrometer to allow electron-capture dissociation (ECD) without the aid of cooling gas or phase-specific electron injection into the cell (Voinov et al., Rapid Commun Mass Spectrom 22, 3087-3088, 2008; Voinov et al., Anal Chem 81, 1238-1243, 2009). Subsequently, we used our rf-free EMS cell in the same instrument platform to demonstrate ECD occurring in the same space and at the same time with collision-induced dissociation (CID) to produce golden pairs and even triplets from peptides (Voinov et al., Rapid Commun Mass Spectrom 23, 3028-3030, 2009). In this report, we demonstrate that ECD and CID product-ion mass spectra can be recorded at high resolution with flexible control of fragmentation processes using a newly designed cell installed in a hybrid Q-TOF tandem mass spectrometer.
\end{abstract}

Key words: Electron capture dissociation, A radio-frequency-free electromagnetostatic cell

\section{Introduction}

$\mathrm{F}$ or ECD to take place efficiently, the precursor ions must 1 comingle with a dense population of low-energy electrons. Because the multiply protonated precursor ions and the reagent electrons have masses differing by up to six orders of magnitude as well as opposite polarities, confining both species in the same volume of space simultaneously is challenging. We have shown this problem could be overcome by guiding the ions with an electrostatic field and focusing the electrons with strong magnetostatic lenses in a linear, rf-free EMS cell built in a Finnigan MAT, San Jose, CA, USA TSQ 700 triple quad mass spectrometer [1-3]. However, the instrument's low mass resolution and mass accuracy precluded analyses of samples typically encountered in proteomics. Consequently, we have installed a newly designed cell in the rf cooling/collision quadrupole section of a Bruker Daltonics, Inc., Billerica, MA, USA,

Correspondence to: Valery Voinov; e-mail: valery.voinov@orst.edu
ultrOTOF-Q hybrid high-resolution mass spectrometer (Figure 1a). The cell (Figure 1b) comprises two axially polarized, $\mathrm{Sm}_{2} \mathrm{Co}_{17}$ disc-magnets (Chino Magnetism, Fairfield, NJ, USA) that have a $76.2 \mathrm{~mm}$ diameter, $7 \mathrm{~mm}$ thickness, and $7 \mathrm{~mm}$ aperture. Soft-iron discs with $2 \mathrm{~mm}$ apertures terminate the entire assembly at both ends. Each iron disc is connected to an independently adjustable $\pm 250-\mathrm{V}$ power supply so that it can function as an element of an electrostatic lens as well as one of a magnetostatic lens. A conically shaped spiral filament of tungsten-rhenium wire of $0.15 \mathrm{~mm}$ diameter (Scientific Instrument Services, Inc., Ringoes, NJ, USA), located concentric with the cell's axis in the center of the cell serves as the source of electrons. This filament surrounds a cylindrical molybdenum grid (HeatWave Labs, Watsonville, CA, USA) with diameter of $1.2 \mathrm{~mm}$; the energy of the electrons entering the reaction zone is the sum of the energy gained by virtue of the potential difference between the filament and grid and by the distribution of energies the electrons have when they are emitted from the filament. In all but one ECD experiment, the filament-grid potential difference was set at $3 \mathrm{~V}$; in one 


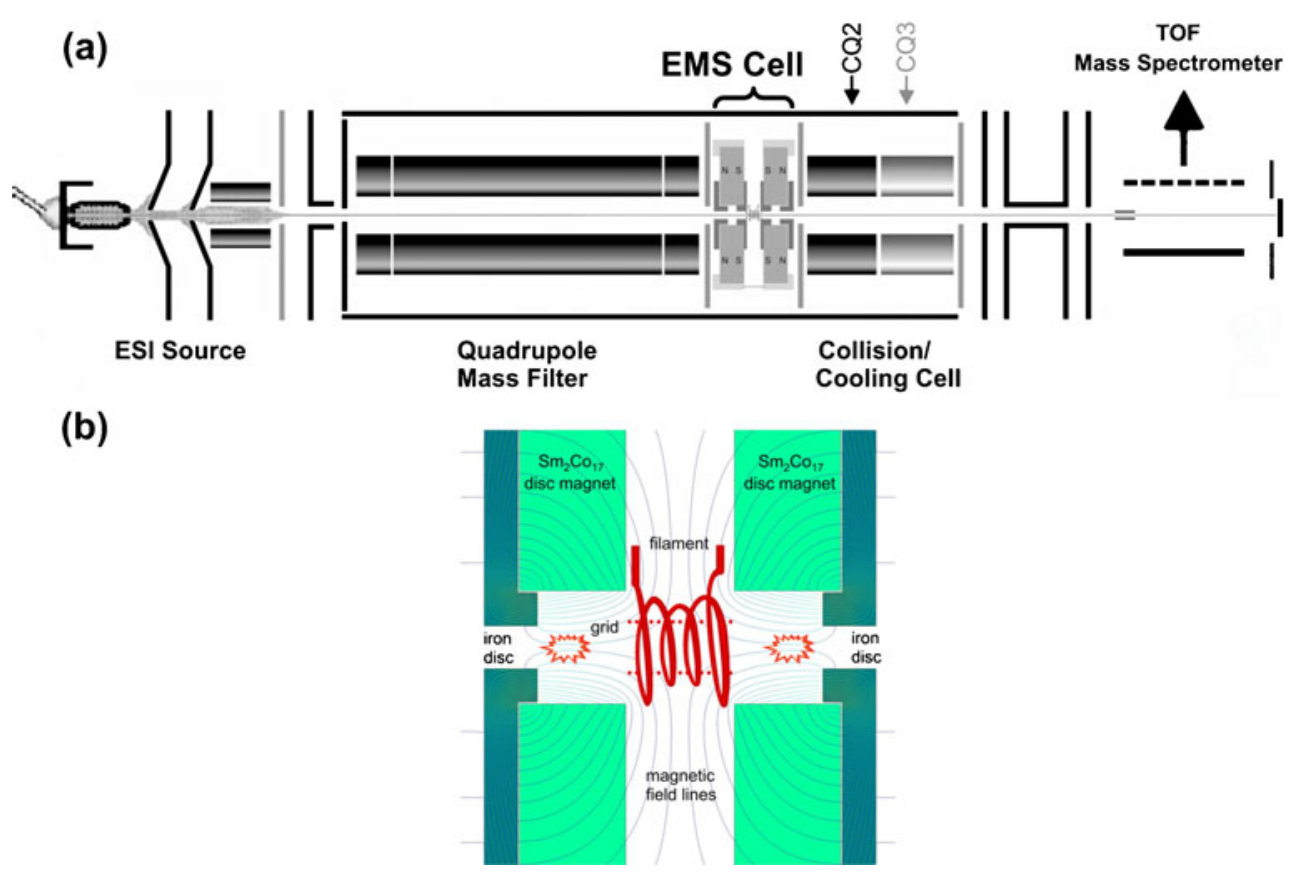

Figure 1. (a) Schematic of the Bruker Daltonics ultrOTOF-Q quadrupole-TOF mass spectrometer showing the retrofitted rf-free EMS ECD/CID cell's location in the segmented collision/cooling multipole section shortened by removing the first segment (CQ1) to accommodate the EMS cell. (b) Cross-section of the EMS cell's interior showing the location and design of the centrally located electron filament and grid assembly

experiment, it was set at $50 \mathrm{~V}$. Electrons emitted from a heated tungsten-rhenium filament of the sort used in this study have thermal energy distributions with spreads $\geq$ $0.7 \mathrm{eV}$ (FWHM) [4]; in the present case, the width of this distribution was increased by about $5 \mathrm{eV}$ due to the drop in voltage across the four to five turns in the filament's spiral. Thus, a large population of electrons with energies below
$1 \mathrm{eV}$ was present in the reaction zone for all except one of the ECD experiments conducted in this study. Collision-gas for CID experiments was admitted into the center of the cell through $1.6 \mathrm{~mm}$ o.d. $\times 0.254 \mathrm{~mm}$ i.d. stainless steel tubing led radially between the cell's two magnets; no provision was made for differentially maintaining the collision gas at a higher pressure in the cell than in the surrounding vacuum.
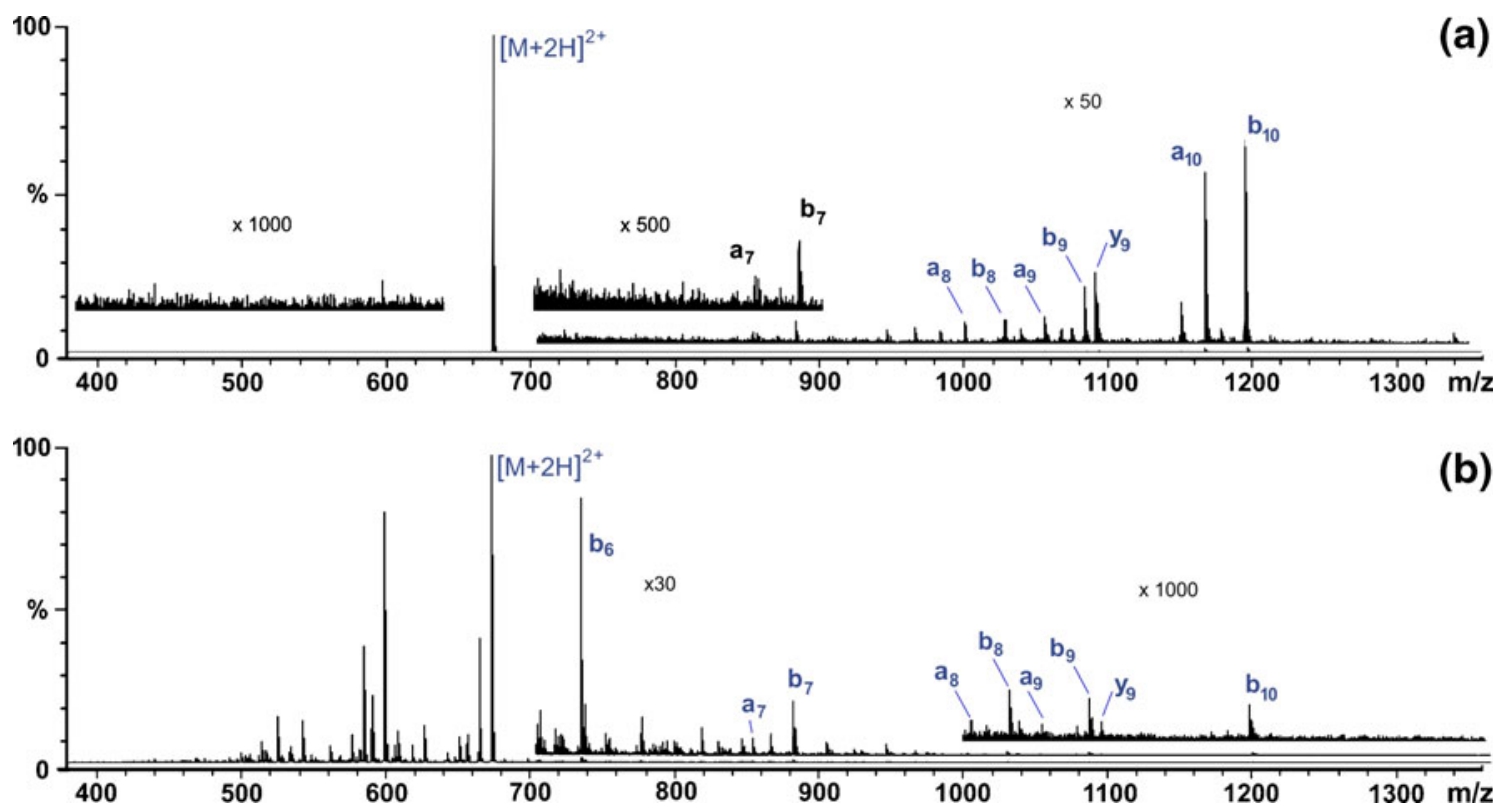

Figure 2. CID product-ion spectra of doubly protonated substance $P(m / z=674)$ : (a) EMS cell, collision gas xenon, collision energy $140 \mathrm{eV}$; (b) shortened rf quadrupole collision/cooling cell, collision gas $\mathrm{N}_{2}$, collision energy $24 \mathrm{eV}$ 
The EMS ECD/CID cell was designed to be operated in various dissociation modes. Using Substance $\mathrm{P}$ as a model compound (American Peptide Co., Sunnyvale, CA, USA), examples of mass spectra recorded in five of the possible combinations are presented: low energy CID, high energy CID, ECD, ECD with collisional excitation in the collisional quadrupole, and ECD in combination with electron ionization decomposition (EID) $[5,6]$.

CID product-ion spectra of doubly protonated Substance P were recorded (1) with xenon (The American Gas Group, Toledo, OH, USA) as the collision gas in the EMS cell and the ion energy (defined relative to the laboratory frame of reference) set at $140 \mathrm{eV}$ (Figure 2a), and (2) with nitrogen as the collision gas in the remaining segments of the original $\mathrm{rf}$ quadrupole collision/cooling cell (CQ2 and CQ3 in Figure 1a) and the ion energy set at $24 \mathrm{eV}$ (Figure 2b). The product-ion spectra produced at the higher collision energy with the EMS cell exhibited signals that increased from low mass to high mass; a similar pattern was observed in CID mass spectra recorded previously with an EMS cell mounted in a Finnigan TSQ 700 triple quadrupole mass spectrometer [3]. The product-ion spectra of Substance P produced at the lower collision energy with the rf quadrupole collision/cooling cell exhibited signals that tended to decrease from low mass to high mass.

ECD product-ion spectra of the doubly protonated Substance P recorded without any sort of excitation, i.e., with an electron energy close to $0 \mathrm{eV}$ and with no simultaneous CID, i.e., via collisions with xenon either in the EMS cell or nitrogen in the collision/cooling quadrupoles (CQ2 and CQ3 in Figure 1a), exhibited characteristic $c$-type fragments whose relative intensities decrease uniformly from low mass to high mass to the point where the molecular cation radical is no longer detectable (Figure 3a). This tendency contrasts strikingly with the nearly uniform distribution of $c$-type fragment ion intensities seen in mass spectra recorded previously with the modified Finnigan TSQ 700 [1]. We suspect that the intensity distribution of $c$-type

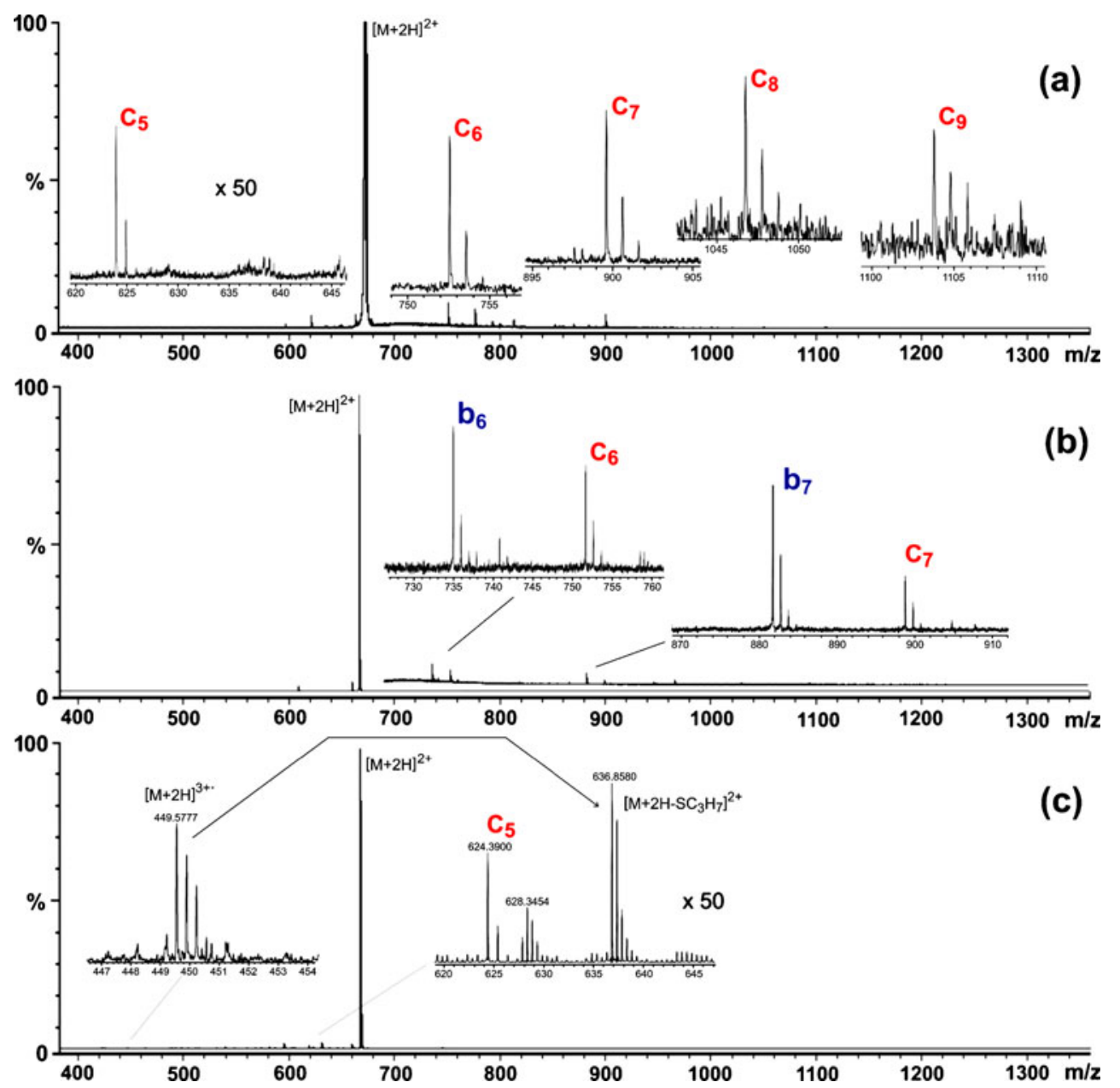

Figure 3. (a) EMS ECD product-ion spectrum of doubly protonated Substance $P(m / z=674)$. (b) EMS ECD/CID product-ion spectrum of doubly protonated Substance P $(\mathrm{m} / \mathrm{z}=674)$. (c) EMS ECD/EID product-ion spectrum of the triply charged radical $[M+$ $2 \mathrm{H}]^{3+\bullet}$ of Substance P; the identity of the radical was ascertained by comparison of the measured mass-to-charge for the monoisotopic signal $(\mathrm{m} / \mathrm{z}=449.578$, resolving power $\sim 13,500$ at $\mathrm{m} / \mathrm{z} 674)$ with the theoretical monoisotopic mass-to-charge $(\mathrm{m} / \mathrm{z}=$ 449.581) 
fragments seen in the spectrum presented in this report (Figure 3a) is a consequence of the relatively long time the $c$-type fragments spend in the instrument's collision/cooling cell (CQ2 and CQ3 in Figure 1a) before being transferred into the orthogonal interface to the TOF mass analyzer (about $400 \mu \mathrm{s}$ ). As shown in earlier experiments ([1] where the time was about $25 \mu \mathrm{s}$ ), a longer residence time will result in a cascade of decomposition of high-mass $c$-type fragments into low-mass $c$-type fragments [7-9], thereby redistributing signal intensities in favor of low-mass $c$-type fragments. This decomposition may have been promoted by collisional excitation that inevitably takes place in the cooling cell.

Mounting the electron filament inside the EMS cell between the magnets resulted in an increase in ECD efficiency by at least an order of magnitude relative to earlier cells [1-3] where the filament was mounted just outside the ion-entry end of the cell. Specifically, the intensity of Substance P's $c_{5}$ fragment was consistently observed in this study to be about $1 \%$ of the precursor-ion intensity (inserts in Figure 3a and c).

Performing ECD with the new cell simultaneously with $\mathrm{CID}$ at $7 \mathrm{eV}$ collisional energy in the remaining segments of the original $\mathrm{rf}$ quadrupole collision/cooling cell (CQ2 and CQ3 in Figure 1a) produced product-ion spectra with golden pairs [10], where mass spectra exhibited both $c$-type (ECD fragments) and $b$-type CID fragments (Figure $3 \mathrm{~b}$ ). Similar results were obtained in our earlier experiments wherein ECD and CID were carried out simultaneously (in time and space) in an EMS cell mounted in a triple quadrupole instrument [3]. This capability could prove useful in topdown analyses of oligopeptides and proteins. At present, CID or IR radiation is used to partially denature such molecules prior to ECD ("activated-ion ECD" [11-13]. An alternative might be to use ECD/CID in an EMS cell with the following multiple low energy collision activation in $\mathrm{rf}$ quadrupole cell as analogous of ECD with following IRMPD [14].

Regions of very high magnetic flux density inside the EMS cell (marked with stars in Figure 1b) dynamically compress large numbers of electrons into a small volume through which the precursor ion beam moves. The electrons in each of these regions propagate in spirals at near thermal energies along directions both opposite to and the same as the direction of the ions through the cell. Consequently, setting the filament at negative potential relative to the grid and magnets results in electrons with energies ranging from $0 \mathrm{eV}$ to a maximum that is determined by the potential difference between the filament and the grid (Figure 1b). Product-ion spectrum shown in Figure 3c was recorded with the filament potential set $50 \mathrm{~V}$ lower than the grid potential, confining electrons with energies from $0 \mathrm{eV}$ to about $50 \mathrm{eV}$ in the cell's reaction spaces. Interactions between doubly protonated Substance P selected as the precursor ion and the low-energy electrons resulted in the appearance of regular $\mathrm{ECD} c$-type fragments in the spectrum. Interactions between doubly protonated Substance P and electrons having energies higher than the precursor's ionization energy resulted in the formation of $[\mathrm{M}+2 \mathrm{H}]^{3+\bullet}$ radical ions. These triply charged radical precursor ions can fragment into ions that are not present in the conventional ECD product-ion spectrum of Substance P. For example, prominent monoisotopic peaks corresponding to $\left[\mathrm{M}+2 \mathrm{H}-\mathrm{SC}_{3} \mathrm{H}_{7}\right]^{2+}$ (theoretical monoisotopic mass $=636.8587$ ) and $[\mathrm{M}+2 \mathrm{H}-$ $\left.\mathrm{SC}_{3} \mathrm{H}_{7}-\mathrm{NH}_{3}\right]^{2+}$ (theoretical monoisotopic mass $=628.3458$ ) appear in the spectrum, respectively, at $m / z=636.8580$ and $m / z=628.3454$ (insert, Figure 3c). These fragments, which have been previously reported [6], are the result of monomolecular decomposition of a $[\mathrm{M}+2 \mathrm{H}]^{3+\bullet}$ radical precursor.

In summary, an EMS ECD cell with an internal electron filament retrofitted into a high performance hybrid quadrupole time-of-flight Bruker ultrOTOF mass spectrometer yielded high mass-resolution, high mass-accuracy ECD product-ion mass spectra that enabled unambiguous identification of fragment ions. This accomplishment supports an earlier assertion [1] that an EMS ECD cell can be operated in various mass spectrometers regardless of analyzer type. In addition, incorporation of an internal electron filament in the cell has made it possible to achieve an ECD efficiency of about $1 \%$ with Substance P. Finally, operating the EMS ECD cell in conjunction with the original $\mathrm{rf}$ collision cell enabled analyses in either a simultaneous ECD/low energy CID mode or an electron ionization/ECD mode. The ECD/ CID mode should complement existing mass spectrometric approaches to top-down analyses of oligopeptides and proteins.

\section{Acknowledgment}

This research was supported directly by grants from the NSF (CHE-0924027), the Oregon Nanoscience and Microtechnologies Institute (\#09-31 \#3.5), NIH (R01RR026275National Center For Research Resources), and the NIEHS (ES00210-Environmental Health Sciences Center). The authors are indebted to Elsworth T. Hinke for his assistance in fabricating the EMS cell used in this study. V.V. thanks Yury Tsybin for helpful discussion. The authors also thank Mel Park and Thomas Knudsen of Bruker Daltonics for their contribution to the work.

\section{References}

1. Voinov, V.G., Deinzer, M.L., Barofsky, D.F.: Electron capture dissociation in a linear radiofrequency-free magnetic cell. Rapid Commun. Mass Spectrom. 22, 3087-3088 (2008)

2. Voinov, V.G., Deinzer, M.L., Barofsky, D.F.: Radio-frequency-free cell for electron capture dissociation in tandem mass spectrometry. Anal. Chem. 81, 1238-1243 (2009)

3. Voinov, V.G., Beckman, J.S., Deinzer, M.L., Barofsky, D.F.: Electroncapture dissociation (ECD), Collision-induced dissociation (CID) and ECD/CID in a linear radiofrequency-free magnetic cell. Rapid Commun. Mass Spectrom. 23, 3028-3030 (2009) 
4. Ryder, J.D.: Electronic fundamentals and applications, 2nd edn, pp. 6681. Prentice-Hall, Inc, Englewood Cliffs (1959)

5. Budnik, B.A., Zubarev, R.A.: $\mathrm{MH}^{2+\bullet}$ ion production from protonated polypeptides by electron impact: observation and determination of ionization energies and a cross-section. Chem. Phys. Lett. 316, 19-23 (2000)

6. Fung, Y.M., Adams, C.M., Zubarev, R.A.: Electron ionization dissociation of singly and multiply charged peptides. J. Am. Chem. Soc. 131(29), 9977-9985 (2009)

7. Leymarie, N., Costello, C.E., O'Connor, P.B.: Electron capture dissociation initiates a free radical reaction cascade. J. Am. Chem. Soc. 125, 8949-8958 (2003)

8. Belyayev, M.A., Cournoyer, J.J., Lin, C., O'Connor, P.B.: The effect of radical trap moieties on electron capture dissociation spectra of substance P. J. Am. Soc. Mass Spectrom. 17, 1428-1436 (2006)

9. Li, X., Cournoyer, J.J., Lin, C., O'Connor, P.B.: The effect of fixed charge modifications on electron capture dissociation. J. Am. Soc. Mass Spectrom. 19, 1514-1526 (2008)
10. Horn, D.M., Zubarev, R.A., McLafferty, F.W.: Automated de novo sequencing of proteins by tandem high-resolution mass spectrometry. Proc. Natl. Acad. Sci. U. S. A. 97, 10313-10317 (2000)

11. Horn, D.M., Ge, Y., McLafferty, F.W.: Activated ion electron capture dissociation for mass spectral sequencing of larger $(42 \mathrm{kDa})$ proteins. Anal. Chem. 72, 4778-4784 (2000)

12. Sze, S.K., Ge, Y., Oh, H., McLafferty, F.W.: Top-down mass spectrometry of a $29-\mathrm{kDa}$ protein for characterization of any posttranslational modification to within one residue. Proc. Natl. Acad. Sci. U. S. A. 99, 1774-1779 (2002)

13. Ge, Y., Lawhorn, B.G., El Naggar, M., Strauss, E., Park, J.H., Begley, T.P., McLafferty, F.W.: Top down characterization of larger proteins $(45 \mathrm{kDa})$ by electron capture dissociation mass spectrometry. J. Am. Chem. Soc. 124, 672-678 (2002)

14. Håkansson, K., Chalmers, M.J., Quinn, J.P., McFarland, M.A., Hendrickson, C.L., Marshall, A.G.: Combined electron capture and infrared multiphoton dissociation for multistage MS/MS in a fourier transform ion cyclotron resonance mass spectrometer. Anal. Chem. 75, 3256-3262 (2003) 